\title{
Association between Abdominal Obesity and Oxidative Stress in Korean Adults
}

\author{
Im Jeong Na, Jae Sun Park, Sat Byul Park* \\ Department of Family Practice and Community Health, Ajou University School of Medicine, Suwon, Korea
}

Background: Obesity causes several changes in the body and is associated with both inflammation and oxidative stress. However, the relationship between abdominal obesity, diacron-reactive oxygen metabolites (d-ROMs), and biological antioxidant potential (BAP) levels in Korean adults has not yet been proved. The aim of this study was to evaluate the association between d-ROMs, BAP levels, and abdominal obesity in the Korean adult population using clinical data.

Methods: A total of 2,367 individuals, aged 18-86 years, were identified from health check-up examination records at a university hospital, between January 2015 and August 2016. Clinical and biochemical parameters, including waist circumference, lipid profile, alcohol drinking status, and smoking status, were investigated. Oxidative stress levels, viz., d-ROM concentration and antioxidant capacity, viz., BAP, were measured.

Results: Subjects with abdominal obesity presented significantly higher levels of d-ROMs compared to those with a normal waist circumference $(\mathrm{P}<0.001)$. After adjusting for age, sex, alcohol drinking status; smoking status; and triglyceride, low-density lipoprotein levels were found to have a significant positive correlation with abdominal obesity $(\mathrm{P}<0.001)$. BAP did not significantly correlate with abdominal obesity.

Conclusion: We observed a positive association between abdominal obesity and d-ROM concentration. This result indicates that abdominal obesity can increase oxidative stress and may affect the pathways involved in obesity, such as the inflammatory pathway. Such correlation analyses were helpful in revealing the causes, as well as methods to reduce, oxidative stress.

Keywords: Abdominal Obesity; Oxidative Stress; Diacron-Reactive Oxygen Metabolites; Biological Antioxidant Potential 


\section{INTRODUCTION}

Obesity, characterized by weight gain caused by excessive fat accumulation, is a global problem ${ }^{1)}$ and considered to be a primary factor in the etiology of several diseases. ${ }^{2)}$ Inflammation and oxidative stress are associated with obesity, ${ }^{3)}$ which induce several changes in the body, such as an inflammatory response. ${ }^{4-7)}$ Moreover, there exists a correlation between oxidative stress and obesity. ${ }^{3)}$ Oxidative stress is caused by an increase in the concentration of reactive oxygen species (ROS) and is responsible for various diseases, such as arteriosclerosis, malignant tumors, and autoimmune diseases. ${ }^{8-12)}$

Diacron-reactive oxygen metabolite (d-ROMs) is a common biomarker that can be used as an index of the products from ROS. d-ROM indicates the level of hydroperoxides, a type of fairly stable reactive oxygen metabolite. ${ }^{13)}$ However, the association of abdominal obesity with d-ROM concentration and biological antioxidant potential (BAP), and its usability as an index of antioxidant potential in Korean adults, has not yet been proved.

The aim of this study was to evaluate the association among dROMs, BAP levels, and abdominal obesity in Korean adults using clinically available data.

\section{METHODS}

\section{Subjects}

Patients aged 18-86 years were identified from health check-up examination records of those admitted to the Ajou University Hospital, Suwon, between January 2015 and August 2016. This study protocol was inspected by an independent Institutional Review Board, which reviewed the ethical issues pertaining to studies on human subjects and provided approval (AJIRB-MED-MDB-18-019).

A total of 2,367 patients (mean age, 53.3 years; $40.0 \%$ women) were investigated. The majority of subjects visited for regular health checkups; thus, did not have serious health problems. The rates of past cardiovascular disease and cancer were $3.34 \%$ and $4.26 \%$, respectively. The examination recorded demographic information (age, sex, smoking, and drinking) and the medical history of subjects. Patients were classified as either non-smoker, ex-smoker, or current smoker based on their smoking status. Patients were also categorized as either nondrinkers or current drinkers, based on their alcohol consumption status. The current drinker category was used for subjects who drank alcohol more than twice a week.

\section{Data Measurement}

According to the results of the 1998 Korea National Health and Nutrition Examination Survey, the reference value for abdominal obesity was defined as $90 \mathrm{~cm}$ in men and $85 \mathrm{~cm}$ in women. ${ }^{14)}$

Clinical and biochemical parameters, including waist circumference and lipid profile, were determined. After overnight fasting, a venous blood sample was obtained the next morning. Each subject fasted for more than 10 hours before blood collection. The serum lipid profile was assayed using a Roche cobas 8000 c702 Automatic Analyzer (Roche Diagnostics Korea, Seoul, Korea).

Serum oxidative stress and antioxidant capacity were measured using automated methods. The oxidative stress level was measured by a d-ROMs test (Diacron S.R.L, Grosseto, Italy), in which the amount of organic hydroperoxide converted into radicals oxidizing N,N-diethylp-phenylenediamine hydroperoxide is measured. ${ }^{9)}$ The results of serum d-ROM levels were expressed in Carratelli units (U.CARR). ${ }^{15-17)}$

In the BAP test (Diacron S.R.L), antioxidant capacity was determined based on the volume of plasma sample required to reduce ferric (Fe 3+) ions to ferrous (Fe 2+) ions. ${ }^{18-20)}$ The results of BAP levels were expressed as $\mu \mathrm{mol} / \mathrm{L}^{15)}$

\section{Statistical Analysis}

Variables are expressed as mean \pm standard deviation (SD). The characteristics of the two groups (abdominal obese group and abdominal non-obese group) were compared using the Student unpaired t-test. After adjusting the comparisons for age, sex, alcohol drinking; smoking status; and total cholesterol (TC), triglyceride (TG), low-density lipoprotein (LDL), and high-density lipoprotein (HDL), the two groups were compared using analysis of covariance.

Statistical analysis was conducted using the IBM SPSS ver. 21.0 software for Windows (IBM Corp., Armonk, NY, USA). A P-value of $<0.05$ was considered to be statistically significant.

\section{RESULTS}

\section{Baseline Characteristics}

The clinical and analytical data (mean $\pm S D$, or subject numbers [percentage]) of the whole group according to abdominal obesity are presented in Table 1. The patients were divided into two groups according to abdominal obesity. The two groups were matched for age; gender; alcohol; smoking; and TC, TG, HDL, and LDL levels. The average age of the subjects was $53.3 \pm 10.5$ years. Of the 2,367 patients investigated in this study, $40.0 \%$ were women. The results showed that alcohol consumption and LDL, HDL, and TG levels were associated with abdominal obesity (P for trend $<0.05$ ) (Table 1 ).

\section{Relationship between Diacron-Reactive Oxygen Metabolite and Biological Antioxidant Potential with Abdominal Obesity}

We observed a positive association between abdominal obesity and $\mathrm{d}$ ROMs ( $\mathrm{P}$ for trend $<0.001$ ). With oxidative stress adjusted for age; sex; smoking; alcohol consumption; and TG, LDL showed a significant positive correlation with abdominal obesity ( $\mathrm{P}$ for trend $<0.001$ ) (Table 2 ).

\section{DISCUSSION}

Our results demonstrated that patients with abdominal obesity presented significantly higher d-ROM levels. Obesity is known to be related to minute chronic systemic inflammation in adipose tissues. This 
Table 1. Participants' characteristics classified as per abdominal obesity

\begin{tabular}{|c|c|c|c|}
\hline Characteristic & Non-abdominal obesity $(n=1,461)$ & Abdominal obesity $(n=906)$ & P-value* \\
\hline Sex (female) & $538(36.8)$ & $427(47.1)$ & $<0.001$ \\
\hline Age $(y)$ & $52.03 \pm 10.33$ & $55.43 \pm 10.42$ & $<0.001$ \\
\hline Waist circumference (cm) & $81.6 \pm 5.2$ & $93.0 \pm 7.2$ & $<0.001$ \\
\hline Diacron-reactive oxygen metabolites (Carratelli units) & $360.5 \pm 74.6$ & $376.4 \pm 75.6$ & $<0.001$ \\
\hline Biological antioxidant potential ( $\mu \mathrm{mol} / \mathrm{L})$ & $2,200.3 \pm 546.2$ & $2,173.4 \pm 272.5$ & 0.112 \\
\hline Alcohol drinking & $837(57.7)$ & $477(52.6)$ & 0.022 \\
\hline Smoking & & & 0.451 \\
\hline Non-smoker & $725(49.6)$ & $463(51.1)$ & \\
\hline Ex-smoker & $426(29.2)$ & $242(26.7)$ & \\
\hline Smoker & $236(16.2)$ & $142(15.8)$ & \\
\hline Total cholesterol (mg/dL) & $200.9 \pm 35.2$ & $205.5 \pm 38.1$ & 0.003 \\
\hline Triglyceride (mg/dL) & $109.0 \pm 83.4$ & $140.7 \pm 87.0$ & $<0.001$ \\
\hline High-density lipoprotein (mg/dL) & $58.1 \pm 15.6$ & $52.5 \pm 12.8$ & $<0.001$ \\
\hline Low-density lipoprotein (mg/dL) & $121.7 \pm 33.0$ & $125.9 \pm 35.9$ & 0.005 \\
\hline Caner history & $59(4.0)$ & $42(4.6)$ & 0.493 \\
\hline Myocardial infarction, angina history & $44(3.0)$ & $34(3.8)$ & 0.332 \\
\hline
\end{tabular}

Values are presented as number (\%) or mean \pm standard deviation.

"P<0.05; by Student unpaired t-test.

Table 2. Independent sampling of the mean difference between d-ROMs levels and BAP with abdominal obesity

\begin{tabular}{|c|c|c|c|c|c|c|}
\hline & \multicolumn{2}{|c|}{ Univariate analysis } & \multirow[b]{2}{*}{ P-value } & \multicolumn{2}{|c|}{ Multivariate analysis } & \multirow[b]{2}{*}{ P-value } \\
\hline & $\begin{array}{l}\text { Non-abdominal obesity } \\
\qquad(\mathrm{n}=1,461)\end{array}$ & $\begin{array}{l}\text { Abdominal obesity } \\
\qquad(n=906)\end{array}$ & & $\begin{array}{l}\text { Non-abdominal obesity } \\
\qquad(\mathrm{n}=1,461)\end{array}$ & $\begin{array}{l}\text { Abdominal obesity } \\
\qquad(\mathrm{n}=906)\end{array}$ & \\
\hline Ln (d-ROMs) & $5.87 \pm 0.21$ & $5.91 \pm 0.20$ & $<0.001$ & $5.86 \pm 0.21$ & $5.91 \pm 0.20$ & $<0.001$ \\
\hline Ln (BAP) & $7.68 \pm 0.15$ & $7.68 \pm 0.13$ & 0.19 & $7.69 \pm 0.15$ & $7.68 \pm 0.13$ & 0.073 \\
\hline
\end{tabular}

Values are presented as mean \pm standard deviation. Multivariate analysis was performed after adjusting for age; sex; smoking status; alcohol use; and triglyceride, low-density lipoprotein, and high-density lipoprotein levels using analysis of covariance.

d-ROM, diacron-reactive oxygen metabolites; BAP biological antioxidant potential.

arises though the inducing triggering of the innate immune system in adipose tissues, which promotes the proinflammatory condition and oxidative stress, giving rise to an acute systemic inflammation. Moreover, several chronic diseases, such as diabetes mellitus, metabolic syndrome, cardiovascular diseases, and cancer, are a result of obesity and also related to oxidative stress. ${ }^{2)}$ Thus, it is inferred that the inflammation of adipose tissues in an obese person plays an important role in the etiology of obesity-related diseases. ${ }^{21)}$

Obesity affects the production of adipokines. ${ }^{5}$ Consequently, blood flow is diminished and hypoxia follows, caused by a progressive increase in the number of adipocytes and amount of adipose tissues. Hypoxia is associated with necrosis and macrophage infiltration into adipose tissues. Infiltrated macrophages form crown-like constructions that surround adipocytes and lead to the overproduction of adipokines and inflammatory mediators, such as interleukin (IL)-6, IL$1 \beta$, and tumor necrosis factor- $\alpha^{5,6)}$ Although C-reactive protein is associated with insulin resistance, IL- 6 has the potential to confuse insulin signal transduction, due to the production of proteins that attach to the insulin receptor. ${ }^{22)}$ At the systemic level, altered adipokine secretion may result in increased food intake and reduced energy expenditure owing to processes in the hypothalamus and decreased liver insulin sensitivity. ${ }^{23)}$
Moreover, necrosis is an additional pathway of cellular apoptosis related to the accumulation of ROS and depends on functional mitochondria. ${ }^{24)}$ Disruption of inter-endothelial junctions between endothelial cells; a significant increase in the production of ROS and inflammation mediators which originate from inflamed endothelial cells; a change in the balance between nitric oxide (NO) synthesis and ROS, insulin signaling, and NO production; and change in L-arginine level, are all associated with endothelial dysfunction during obesity. ${ }^{25)}$

A limitation of our study is that we voluntarily analyzed data from participants who visited the health promotion center. Thus, our study group may not represent the general population. Secondly, other oxidative stress measurement methods are also available, but we used the measurement method used by Ajou University Hospital. This method has the advantage of being able to obtain a lot of data with ease, but is less stable compared to other technologies. Another limitation is the lack of research on other factors that affect oxidative stress. Regardless of these limitations, our study is valuable because it is the first to evaluate the importance of the association between oxidative stress and abdominal obesity. Another advantage of this study is that it provides insights on the effect of antioxidants for obesity-related diseases.

In conclusion, we observed a positive association between abdominal obesity and d-ROMs. The present analysis shows that abdominal 
obesity can increase oxidative stress and may affect the development of the pathways involved in obesity, such as the inflammatory pathway. The results of this study are helpful in revealing the cause of oxidative stress and methods to reduce oxidative stress.

\section{CONFLICT OF INTEREST}

No potential conflict of interest relevant to this article was reported.

\section{ORCID}

Im Jeong Na: https://orcid.org/0000-0002-1627-5952

Jae Sun Park: https://orcid.org/0000-0003-1536-0271

Sat Byul Park: https://orcid.org/0000-0003-1090-716X

\section{REFERENCES}

1. Sikaris KA. The clinical biochemistry of obesity. Clin Biochem Rev 2004;25:165-81.

2. Alberti KG, Zimmet PZ. Definition, diagnosis and classification of diabetes mellitus and its complications. Part 1: diagnosis and classification of diabetes mellitus provisional report of a WHO consultation. Diabet Med 1998;15:539-53.

3. Bondia-Pons I, Ryan L, Martinez JA. Oxidative stress and inflammation interactions in human obesity. J Physiol Biochem 2012;68:701-11.

4. Maury E, Brichard SM. Adipokine dysregulation, adipose tissue inflammation and metabolic syndrome. Mol Cell Endocrinol 2010;314: 1-16.

5. Galic S, Oakhill JS, Steinberg GR. Adipose tissue as an endocrine organ. Mol Cell Endocrinol 2010;316:129-39.

6. Johnson AR, Milner JJ, Makowski L. The inflammation highway: metabolism accelerates inflammatory traffic in obesity. Immunol Rev 2012;249:218-38.

7. Mraz M, Haluzik M. The role of adipose tissue immune cells in obesity and low-grade inflammation. J Endocrinol 2014;222:R113-27.

8. Aviram M. Review of human studies on oxidative damage and antioxidant protection related to cardiovascular diseases. Free Radic Res 2000;33 Suppl:S85-97.

9. Kamezaki F, Yamashita K, Kubara T, Suzuki Y, Tanaka S, Rkouzuma R, et al. Derivatives of reactive oxygen metabolites correlates with highsensitivity C-reactive protein. J Atheroscler Thromb 2008;15:206-12.

10. Katsabeki-Katsafli A, Kerenidi T, Kostikas K, Dalaveris E, Kiropoulos TS, Gogou E, et al. Serum vascular endothelial growth factor is related to systemic oxidative stress in patients with lung cancer. Lung Cancer 2008;60:271-6.

11. Kotani K, Koibuchi H, Miyamoto M, Yamada T, Taniguchi N. Relation- ship between reactive oxygen metabolites and carotid intima-media thickness in subjects with hypercholesterolemia. Med Princ Pract 2010;19:496-8.

12. Sakane N, Fujiwara S, Sano Y, Domichi M, Tsuzaki K, Matsuoka Y, et al. Oxidative stress, inflammation, and atherosclerotic changes in retinal arteries in the Japanese population; results from the Mima study. Endocr J 2008;55:485-8.

13. Martinovic J, Dopsaj V, Kotur-Stevuljevic J, Dopsaj M, Vujovic A, Stefanovic A, et al. Oxidative stress biomarker monitoring in elite women volleyball athletes during a 6-week training period. J Strength Cond Res 2011;25:1360-7.

14. Lee SY, Park HS, Kim DJ, Han JH, Kim SM, Cho GJ, et al. Appropriate waist circumference cutoff points for central obesity in Korean adults. Diabetes Res Clin Pract 2007;75:72-80.

15. Pasquini A, Luchetti E, Marchetti V, Cardini G, Iorio EL. Analytical performances of d-ROMs test and BAP test in canine plasma: definition of the normal range in healthy Labrador dogs. Vet Res Commun 2008;32: 137-43.

16. Cesarone MR, Belcaro G, Carratelli M, Cornelli U, De Sanctis MT, Incandela L, et al. A simple test to monitor oxidative stress. Int Angiol 1999;18:127-30.

17. Trotti R, Carratelli M, Barbieri M. Performance and clinical application of a new, fast method for the detection of hydroperoxides in serum. Panminerva Med 2002;44:37-40.

18. Fukui T, Yamauchi K, Maruyama M, Yasuda T, Kohno M, Abe Y. Significance of measuring oxidative stress in lifestyle-related diseases from the viewpoint of correlation between d-ROMs and BAP in Japanese subjects. Hypertens Res 2011;34:1041-5.

19. Dohi K, Satoh K, Ohtaki H, Shioda S, Miyake Y, Shindo M, et al. Elevated plasma levels of bilirubin in patients with neurotrauma reflect its pathophysiological role in free radical scavenging. In Vivo 2005;19: $855-60$.

20. Moriarity JT, Folsom AR, Iribarren C, Nieto FJ, Rosamond WD. Serum uric acid and risk of coronary heart disease: Atherosclerosis Risk in Communities (ARIC) study. Ann Epidemiol 2000;10:136-43.

21. Xu H, Barnes GT, Yang Q, Tan G, Yang D, Chou CJ, et al. Chronic inflammation in fat plays a crucial role in the development of obesity-related insulin resistance. J Clin Invest 2003;112:1821-30.

22. Pradhan AD, Manson JE, Rifai N, Buring JE, Ridker PM. C-reactive protein, interleukin 6 , and risk of developing type 2 diabetes mellitus. JAMA 2001;286:327-34.

23. De Mello AH, Costa AB, Engel JDG, Rezin GT. Mitochondrial dysfunction in obesity. Life Sci 2018;192:26-32.

24. Rockenfeller P, Ring J, Muschett V, Beranek A, Buettner S, CarmonaGutierrez D, et al. Fatty acids trigger mitochondrion-dependent necrosis. Cell Cycle 2010;9:2836-42.

25. Engin A. Endothelial dysfunction in obesity. Adv Exp Med Biol 2017; 960:345-79. 